trotz aller Beschränktheit, Wichtigkeit und Sinn zugeschrieben werden kann. Und so lässt sich hier mit einem Satz aus $\S 402$ und einem Horaz-Zitat aus $\S 403$, die sich in Baumgartens Ästhetik finden, schließen:,Eine groteske Arroganz liegt gar darin, wenn jemand eingesteht, dass er mit kleineren Schiffen keine Erfahrung hat, sich aber auf die Lenkung von Fünfruderern oder von Schiffen, die noch größer sind, verstehen will." Und weiter in $\S$ 403: „Gleichwohl das für erhabene Dinge genügend große Gemüt, nicht das jenes weisen Stoikers ist, den, wenn zerborsten zusammensinkt das Weltall - unerschrocken die Trümmer treffen, dennoch weder von kleineren Sorgen geplagt, noch seiner ruhigen Heiterkeit beraubt, die das Leben der Götter nachahmt.“"

\title{
Zwischen Pathos und Leidenschaft
}

\author{
Von ANDREAS BRENNER (Basel)
}

\author{
RICHARD SHUSTERMAN: LEIBLICHE ERFAHRUNG IN KUNST UND LEBENSSTIL. \\ Akademie Verlag, Berlin 2005, 208 S.
}

STEFAN NOWOTNY / MICHAEL STAUDIGL: PERSPEKTIVEN DES LEBENSBEGRIFFS. Randgänge der Phänomenologie. Georg Olms Verlag, Hildesheim 2005, 378 S.

Kann denn leibliche Schönheit Sünde sein? Nicht nur eine durch Augustinus geprägte christliche Tradition will diese Frage bejahen, auch die Ästhetik seit der Aufklärung hält von der Schönheit, sofern sie in der Gestalt des menschlichen Körpers sich offenbart, wenig. Und der bis in unsere Tage anhaltende Körperkult bestätigt demnach lediglich die philosophischen Vermutungen, dass der Körper die niederen und weniger schönen Anteile des Menschen enthalte, wahre Schönheit aber in deren Überwindung und mithin in der Überhöhung des Menschen über seine eigene Natur zu erlangen sei.

Mit dieser Zustandsbeschreibung, für die Shusterman in der Moderne sowohl die Ästhetik Baumgartens als auch die Gesellschaftskritik von Horkheimer und Adorno verantwortlich macht, zeichnet er in schnellen Zügen das Bild einer bis in ihre Grundfesten ausgemergelten Kultur, die zum Scheitern verurteilt ist, wenn es ihr nicht gelingt, ihren reduktionistischen Starrkrampf zu lösen und den Menschen zu seiner eigenen Sinnlichkeit freizulassen. Das Heilmittel, das Shusterman in dieser Lage empfiehlt, trägt den Namen Somästhetik. In dem vorliegenden Buch, das eine für die deutsche Übersetzung stark erweiterte Fassung von Performing Life darstellt, geht es Shusterman darum, die Segnungen dieser Medizin zu preisen und nebenbei auch ein wenig Werbung für ihren Erfinder zu machen.

Doch zunächst einmal zum Mittel selbst. Um was geht es? Wie bereits der Begriff verrät, geht es um eine Zusammenführung von aisthesis und soma, also der Wahrnehmung und des Leibes. Bereits diese Begriffskonjunktion wirft einige Fragen auf: Sollen wir also den Leib wahrnehmen, oder sollen wir mit dem Leib wahrnehmen? Und davon abgesehen: Warum sollten wir überhaupt ,,somatisch“ wahrnehmen?

1 A. G. Baumgarten, Ästhetik, Teil I, §§ 1-613, Hamburg 2007, 381. 
Dies sollen wir aus keinem geringeren Grunde als jenem, dem, wie Shusterman erklärt, in der Philosophie die größte Bedeutung zugemessen wird: „Wissen, Tugend und das gute Leben.“ Damit ist der Kaufanreiz für das Mittel Somästhetik deutlich gemacht, es verhilft uns also zu den genannten drei begehrten Gütern. Inwiefern sich diese mittels Somästhetik einstellen, erklärt der Autor allerdings nicht weiter und führt stattdessen aus, was uns alles entgeht, wenn wir den Körper beziehungsweise den Leib gering schätzen. Die Verlustbilanz kann man dabei, wie Shusterman erklärt, zweiteilen, in darstellungsorientierte und erlebnisbezogene Formen. Zu den Ersteren zählen demnach unter anderem das Make-up, das Frisieren und die plastische Chirurgie, zu den erlebnisbezogenen Formen zählen Körpererfahrungen wie Yoga, Feldenkrais-Methode oder die Alexander-Technik.

Hat man diesen Beipackzettel gelesen, mag man sich fragen, ob denn in unserem Mittel der Somästhetik auch Philosophie darinnen ist. Und wie, belehrt uns deren Erfinder: Denn ,die konkrete Aktivität der Beschäftigung mit dem Körper" zielt auf die ,philosophische Disziplin Selbsterkenntnis und Selbstsorge“". Wem dies noch nicht genügt, der wird daran erinnert, dass unser Körper mehr ist als nur ein Gegenstand unseres Stylings, dass er doch schließlich der „lebendige Ort schöner, persönlicher Erfahrungen" sei. Und damit ist Shusterman bei seinem Thema angekommen, das er bereits vor einigen Jahren in Philosophie als Lebenspraxis ausgearbeitet hat, wo auch der Begriff der Somästhetik eingeführt wurde. Im vorliegenden Buch wird dieser Begriff nun stärker in seiner praktischen Bedeutung artikuliert. Wie damals, so bleibt auch jetzt unklar, was Shusterman unter dem Begriff soma verstanden wissen will. Mal scheint es, als verstünde er ihn als jenes dem äußeren Anschein Zugängliche, womit er sich also auf das in der platonischen Tradition Stehende und zum lateinischen corpus Gewandelte bezöge, mal erweckt er den Eindruck, als wollte er ein tieferes und fülligeres Verständnis des menschlichen Körpers einführen. Für die erste Version sprechen alle von Shusterman vorgeschlagenen Weisen der Stilisierung unseres Körpers, für die andere spricht die Bedeutung, die Shusterman dem Körper für die Gestaltung des Selbst zuspricht. Die Differenz, die hier aufgemacht, wenn auch nicht begründet wird, ist die von Körper und Leib.

Da Shusterman diesen Unterschied nicht herausarbeitet, kippt sein Programm der Somästhetik im Zweifel hin zur Körpertherapie. Somästhetik erweist sich damit medizinisch als Placebo und taugt bestenfalls als Kosmetikum.

Die kulturkritische Note, die Shusterman dem abendländischen Denken in Sachen Körpervergessenheit, also eigentlich „Leibvergessenheit“ erteilt, erweist sich jedoch als in der Sache berechtigt, indes verlangt dies nach einer qualifizierteren Begrifflichkeit.

Was man bei Shusterman vermisst, findet man in dem Sammelband von Nowotny und Staudigl, der sich mit dem Werk des französischen Phänomenologen und Leibphilosophen Michel Henry (1922-2002) beschäftigt. Trotz offensichtlicher Parallelen zu dem vorgenannten Buch könnte der Kontrast kaum größer ausfallen. Der, fast möchte man sagen, heilige Ernst, mit dem hier das Werk Henrys analysiert wird, der auch als Theologe hervorgetreten ist und dessen Bücher sich unter ebenso schlichten wie gewichtigen Titeln wie Ich bin die Wahrheit oder Inkarnation in Position bringen, hat etwas Erfrischendes.

Zum Verständnis Henrys sei vorangeschickt, dass er die in der Philosophie zu verzeichnende Leibvergessenheit nicht erst im cartesischen Dualismus, sondern bereits in der platonischen Aufspaltung der Welt festmacht. Bereits hier fällt der Klassenunterschied zu Shusterman auf: Während dieser die mangelnde philosophische Würdigung von Körpertechnologien beklagt und damit bestenfalls die Spitze des Eisberges markiert, sucht Henry den Grund. Wer, wie Shusterman, die Körpervergessenheit des dominanten Teils der Philosophie beklagt, hat das eigentliche Problem noch gar nicht gesehen. Denn wenn man von einer Krise der abendländischen Kultur reden möchte, dann zeigt sich diese wohl kaum darin, dass Körper- 
technologien nicht die nötige Achtung entgegengebracht wird - was, nebenbei bemerkt, auch kaum der Realität der konsumorientierten Gegenwart entspricht. Das eigentliche Problem liegt tiefer: Die Philosophie hat weitgehend die Fähigkeit verloren, menschliche Körper anders als bloß körperlich zu denken.

Wie Hermann Schmitz, der andere Leibphilosoph von überragendem Rang, geht Henry davon aus, dass das Aufkommen des Rationalismus den Zugang zum Leib verdeckt hat. In Kontrast zu Schmitz anerkennt Henry das Christentum als die Kultur, welche den Gedanken an den Leib bewahrt hat. Das Christentum hat demzufolge die Scheidung von Seele (Geist) und Körper nicht mitvollzogen und denkt beides vereint im Begriff des Leibes. Zum Verständnis dieses Zusammenhangs und zugleich der Bedeutung von „Inkarnation“ zitiert Henry denn auch das Johannes-Wort: „Und das Wort ist Fleisch geworden.“ (Joh. 1, 14) Mit dieser Offenbarung hat Henry zufolge das Christentum den Dualismus des Rationalismus überwunden und zugleich das Andere des Körpers als denkbar belassen. Da, wo der Rationalismus nur Körper erkennt, weiß das Christentum um das Fleisch (französisch la chair). Unbesehen seines theologischen Stellenwertes kann man in dem Johannes-Wort auch eine anthropologische Aussage erkennen: Der Mensch ist Fleisch.

Als Fleisch ist der Mensch durch die Spannweite seiner Erfahrungen ausgewiesen, die das menschliche Leben ausmachen. Fleischlich lassen sie sich begreifen und nur fleischlich verstehen wir mithin den Menschen. Dies gilt sowohl für die Geburt und das Wachsen des Menschen als auch für sein Altern. In diesen existenziell verdichteten Phasen zeigt sich, wie Michael Staudigl in seinem Beitrag über Das alternde Leben schreibt, dass Fleisch „verletzlich“ ist. „Seine Verletzlichkeit aber besteht darin“, wie Staudigl fortfährt, dass ihm das Leben genommen werden kann. Das Leben kann dem Fleisch auf vielfältige Weise genommen werden, ohne dass ihm damit zugleich und zur Erlösung der Tod gegeben würde. Phänomenologie macht also, wie Staudigl zeigt, nicht nur die Gabe, sondern auch ihr Gegenteil, das Nehmen oder die Mitnahme, denkbar. Fleisch zeigt sich genau in diesem Dazwischen, es ist Gabe und leidet Mitnahme, wofür wir die Bezeichnung Alter haben. Wenn Fleisch Leben ist, nimmt uns das Altern Leben, ohne es aber zugleich aufzuheben. Dass wir, solange wir altern, leben, macht das Paradox unserer Existenz aus, die sich in permanenter Mitnahme erhält. Wir sind also Zeugen dieser, wie Staudigl formuliert, „Ent-eignung des Lebens“. Dieses Ereignis spielt sich in der Zeit ab und ist als zeitliches fassbar, wie dies Emmanuel Lévinas in seiner Philosophie des Alterns ${ }^{1}$ beschrieben hat, die Staudigl mit Henry ins Gespräch bringt. Der temporäre Aspekt des Alterns wird jedoch erst in der leiblichen Selbstaffektion erfahrbar. Altern ist also nur als fleischliche Veränderung erlebbar, wobei auch hier wiederum ein Topos von Lévinas aufscheint: Diese Veränderung hat eine Alteritäts-Dimension, indes in der Weise, das hier der Andere in mir erlebbar wird. Solche Alterungserlebnisse gehen einher mit der am eigenen Fleisch gespürten und einen verstörenden Erfahrung der Selbstbefremdung.

Verstörend sind Alterungserlebnisse, aber nicht nur diese, deshalb, weil die Leiblichkeit des Menschen, sein Fleisch also, den Menschen voll und ganz ausmacht. Im normalen Vollzug seines Daseins hat der Mensch keine Distanz zu seinem Fleisch, er ist identisch mit seinem Fleisch, er ist sein Fleisch. Fremd wird man sich also erst: Fleischlichkeit müssen wir uns, wie Jean-Michel Longeneaux in seinem Beitrag Das „Fleisch der Welt" ausführt, als eine Unmittelbarkeit vorstellen, zu der wir allgemein kein relationales Verhältnis haben. Es sind die im wahrsten Sinne einschneidenden Erfahrungen, die einen zu ,seinem“ Leib in Beziehung setzen. Die vielleicht drastischste Erfahrung, die uns in Bezug zu uns bringt, ist die der „Berührung“. Henry hat die Husserlsche Beobachtung der Doppelempfindung,

\footnotetext{
E. Lévinas, Jenseits des Seins oder anders als Sein geschieht, Freiburg 1998.
} 
welche diesem zur Entdeckung des Leibes diente, in seiner Untersuchung der „Berührung“ ausgebaut. Entsprechend gilt auch für die Berührung der Doppelaspekt, sowohl Aktives als auch Passives in sich zu fassen. Diese Erfahrung ist unabhängig von ihrer moralischen Güte zu beobachten; sie gilt für den seinen Patienten abtastenden Arzt ebenso wie für den Hand an sein Opfer legenden Folterer, und sie ist auch im erotischen Erleben präsent. Immer dann gerät der Mensch an seine Grenze, und die Grenze des Menschen ist die Grenze seines Leibes, also seine Haut. Henry nennt sie ,jene sichtbare und unsichtbare Linie, auf welcher sich sowohl unsere kinetischen Empfindungen sowie auch diejenigen unserer Sinnesherkunft verknüpfen".?

Longenaux versteht diese Erfahrungen als Prozesse der Selbstaffirmation. Damit deutet er Henry nicht falsch, aber verkürzt. „Selbstaffirmation“ ist eine allzu schwache, weil ausgedünnte Bezeichnung für eine Erfahrung heiliger Dimension. Da das Leben des Fleisches, wie unter anderem die Erfahrung des Alters zeigt, Pathos ist, Pathos aber, wie wir an den Erfahrungen der Berührung gesehen haben, den Leib offenbart, so kann man, wie es Michel Henry in seiner „Philosophie des Leibes“ tut, die schmerzhafte Leibwerdung des Menschen als seine Parusie bezeichnen.

Nun wird auch deutlich, dass Henry mit der Theologie über diese hinausgeht und seine Philosophie des Leibes als eine Anthropologie anlegt. Spätestens hier wird sich wohl auch die Kritik an Henry zu Wort melden. Ist der Leib nur um den Preis der Transzendenz - und dieser Transzendenz - zu denken? Die Leibphilosophie von Schmitz lehnt diesen Weg bekanntlich ab und macht stattdessen das affektive Betroffensein des leiblichen Menschen stark, was sich als ein weniger voraussetzungsreicher und damit als ein weniger beschwerlicher Weg erweist.

Gleichwohl ist auch Henrys Leibphilosophie insofern lebenstauglich, als sie sich beispielsweise auch auf die Phänomene von Krankheit (der Beitrag von Joachim Widder) und Bioethik (Stefan Nowottny) anwenden lässt. Eine qualifizierte Theorie des Leibes, das zeigt dieser Band, gründet das menschliche Selbstverständnis in einer Tiefe, von der aus Fragen nach der körperlichen Selbststilisierung in ihrer Bedeutsamkeit verblassen.

2 M. Henry, Inkarnation. Eine Philosophie des Fleisches, Freiburg 2002, 257. 
Brought to you by | Universitaetsbibliothek Basel

Authenticated Download Date | 10/19/17 12:08 PM 